\title{
Tropical sprue presenting with dementia
}

\begin{abstract}
Celiac and Tropical Sprue are malabsorptive diseases with similar clinical manifestations and histological findings. While, celiac sprue is a known cause of malabsorption in the Western world, Tropical sprue is relatively uncommon and often overlooked by the medical community. Misdiagnosis can result in delay of adequate treatment in a patient with Tropical Sprue. We present a patient with a Tropical Sprue, who initially diagnosed with an iron-deficient anemia and dementia. This case aims to increase the awareness of gastroenterologists, who are likely to encounter patients from different parts of the world, due to todays' globalization.
\end{abstract}

Keywords: tropical sprue, dementia, anemia

\author{
Volume 2 Issue 4 - 2015 \\ Shalini Jain,' Ahmed Bedeir, ${ }^{2}$ Esra Nutku \\ Bilir, Bahri Bilir ${ }^{4}$ \\ 'University of Colorado, USA \\ ${ }^{2}$ Miraca Life Sciences, USA \\ ${ }^{3}$ Scientific Director, USA \\ ${ }^{4}$ Rocky Mountain Gastroenetrology, USA
}

Correspondence: Bahri Bilir, Rocky Mountain

Gastroenterology Arapahoe Endoscopy, 1001 South park Drive, Littleton, CO., 80I20, USA, Tel 720-933-8806,

Emailbmbilir@yahoo.com

Received: July 09, 2015 | Published: August 14, 2015

\section{Introduction}

Tropical Sprue is an uncommon cause of small bowel malabsorption, in the Western world, and often overlooked by the medical community. Misdiagnosis can result in delay of adequate treatment in a patient with this illness. ${ }^{1-3}$ A 61 -year old, originally Nepalese, female patient was sent as a direct referral for an EGD and a colonoscopy for severe "iron deficiency anemia" and a positive FIT test. She presented with a complaint of a $10-1 \mathrm{~b}$ weight loss, heme positive stool, dyspepsia and a decrease in appetite. The patient spent 10years in a refugee camp in Bhutan before she immigrated to the U.S. In last 10years, she had significant decline of her energy levels, appetite, and cognitive functions and was diagnosed presumptively with early onset Alzheimer's disease. She complained of chronic constipation with intermittent diarrhea every few days, and epigastric burning aggravated with spicy foods. She was given ranitidine, which did not change her complaints. She denied dysphagia, nausea/vomiting, fever, sick contacts, melena or hematochezia. Despite the interpreter's involvement and clear instructions given about the colonoscopy prep, the patient could not follow the instructions due to her significant memory problems. Her initial physical exam was unremarkable other than a faint murmur in all regions of her chest. Pertinent lab results were: H. Pylori stool antigen: negative; stool O \& P: negative; WBC: $4,100 / \mathrm{uL}$ with a mild lymphocytosis of $51 \%$, hemoglobin: $9.5 \mathrm{~g} / \mathrm{dL}$, hematocrit: $27.1 \%$, MCV: $107 \mathrm{fL}$, RDW: $15.6 \%$, platelets: 105,000/ uL. Comprehensive metabolic panel was normal except for BUN: 7mg/dL; ALT: $34 \mathrm{IU} / \mathrm{L}$, and TSH: 2.730uIU/ml. An upper endoscopy revealed mild inflammation of the gastric antrum. Flattening and scalloping of the duodenal folds were noted in the entire duodenum, along with some thickening, typical for celiac sprue (Figures1 \&v2). ${ }^{4}$ The low prevalence of celiac sprue in the Nepalese population, and patient's years spent in Southeast Asia raised the possibility of tropical sprue as a diagnosis associated with malabsorption and reversible dementia. Due to her atypical presentation, vitamin $\mathrm{B}_{12}$, folic acid and thiamine levels were ordered. To exclude celiac disease, HLADQ testing was performed. The results showed that her DQ2 was positive, and DQ8 negative. Her colonoscopy was normal throughout including the terminal ileum. Random biopsies of the colonic mucosa were negative for microscopic colitis. Additional tests revealed: low vitamin $\mathrm{B}_{12}(31 \mathrm{pg} / \mathrm{mL})$; Folic acid: $9.1 \mathrm{ng} / \mathrm{mL}$; t-Transglutaminase (tTG) $\operatorname{IgA}<2 \mathrm{U} / \mathrm{mL} ; H$. pylori was positive on the gastric biopsy results.

Based on the results, the patient was treated for with clarithromycin, pantoprazole, metronidazole and amoxicillin. For atypical sprue and possible tropical sprue, sulfamethoxazole and trimethoprim was prescribed for the next three months. After the treatments, vitamin $\mathrm{B}_{12}$ and two multivitamins daily were prescribed. Gluten-free diet was not prescribed. The patient returned 3 months later for an upper endoscopy. She had improved energy, cognitive function, and she looked a lot younger by people who knew her. The repeat EGD showed only mild duodenopathy without changes consistent with celiac sprue (Figure 3). Follow up duodenal biopsy showed signs of improvement including partial villous regeneration and decrease of intraepithelial lymphocytes (Figure 4). Follow up testing revealed: WBC 4.4k/ uL; HGB: 10.7g/dL; HCT: 31.9\%; MCV: 86fL; Platelets: 194 k/uL; Ferritin: 122ng/mL; ALT: 26U/L; AST: 20U/L; Iron: 80ug/dL; TIBC: $275 \mathrm{ug} / \mathrm{dL} ; 29 \%$ saturation. Vitamin $\mathrm{B}_{12}: 104 \mathrm{pg} / \mathrm{mL}$; Folic acid $>20 \mathrm{ng} /$ $\mathrm{mL}$ She was treated with sulfamethoxazole and trimethoprim for next three months.

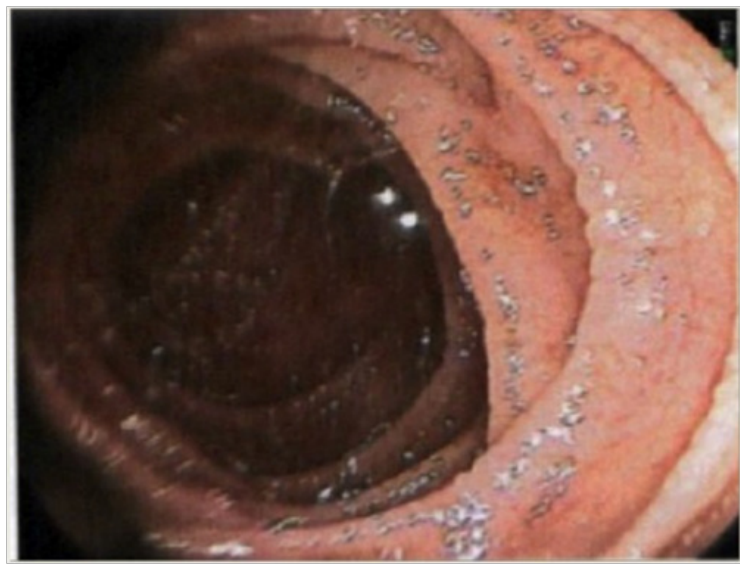

Figure I Initial endoscopy showing scalloping of duodenal folds, typical for Tropical and/ or Celiac Sprue. 


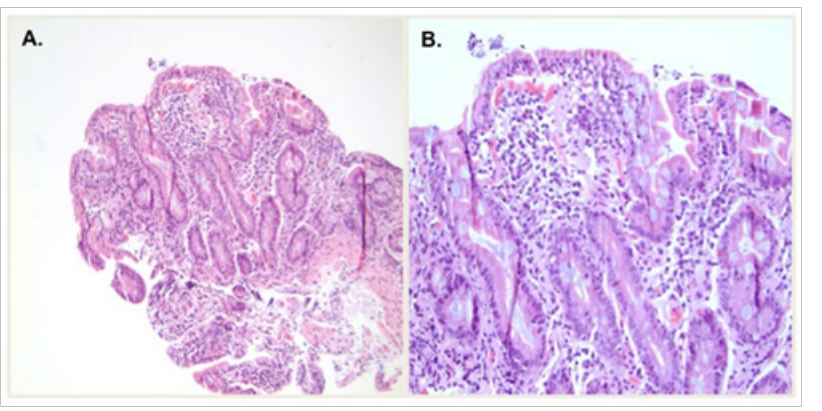

Figure 2 First duodenal biopsy showing severe villous blunting, crypt hyperplasia with marked increased intraepithelial lymphocytes and epithelial degeneration. (A) Low power (I0x); (B) high power (20x).

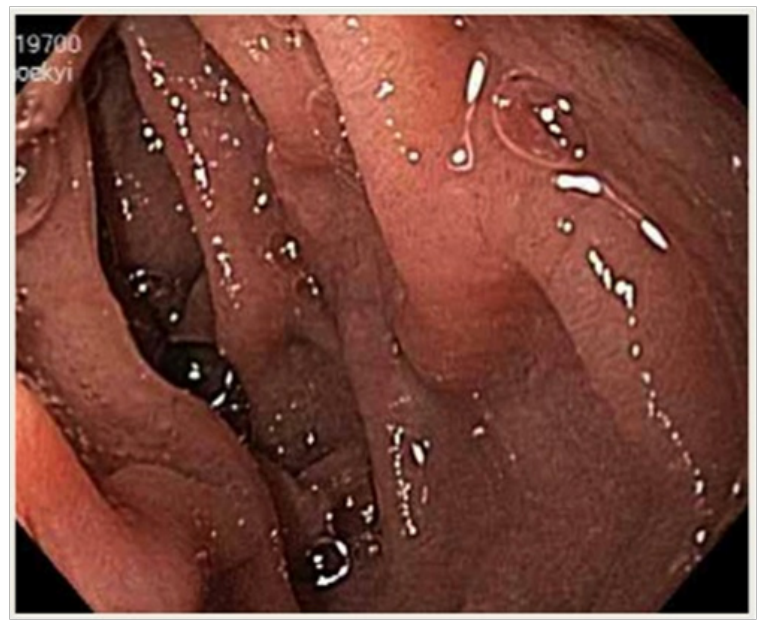

Figure 3 Mild duodenopathy without changes consistent with celiac sprue.

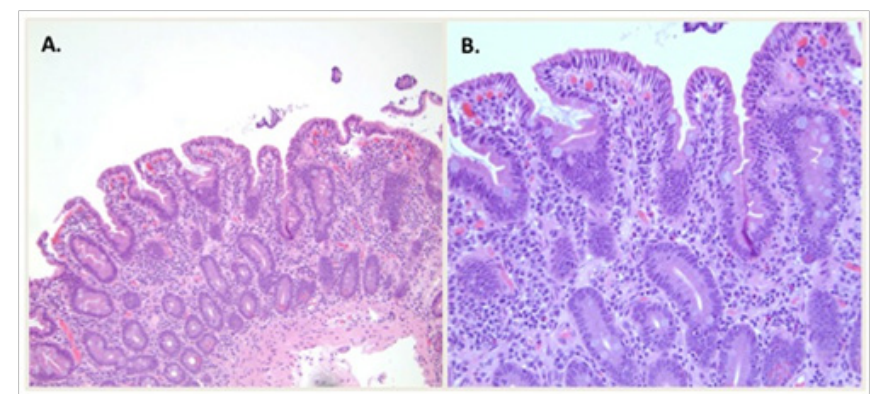

Figure 4 Follow up duodenal biopsy showing signs of improvement including partial villous regeneration and decrease of intraepithelial lymphocytes. (A) Low power (10x); (B) High power (20x).

\section{Discussion}

Tropical sprue is caused by inflammation and damage to the small intestine from a suspected yet unidentified bacterial infection. The inflammation causes malabsorption of nutrients due to the increased swelling of the small intestine. This condition is common in patients who have lived or visited tropical places for extended periods of time.
Tropical sprue may not develop until after the patient has left the tropical area, lagging even up to 10 years. It is still seen in Southeast Asia and the Caribbean, excluding Jamaica. There is no associated specificity for race, gender or age. ${ }^{1,3}$

The most common symptoms of tropical sprue are abdominal cramps, diarrhea, indigestion, irritability, muscle cramps, numbness, and weight loss. Malabsorption of iron, folate, vitamin $\mathrm{B}_{12}$ and associated deficiencies of vitamins A, D and Kare also common. Laboratory test abnormalities may include but not limited to are as follows: Macrocytic anemia with low folate and vitamin $\mathrm{B}_{12}$ levels; low levels of serum potassium, iron and albumin; increased levels of urea; abnormal calcium and phosphate levels; quantitative 24-hour fecal fat collection of over $6 \mathrm{~g}$; duodenal/ jejunal biopsies revealing incomplete villous atrophy. Differential diagnoses include secondary malabsorption due to helminthic, protozoal, bacterial, or viral infections, Crohn's disease, tuberculosis, pancreatic insufficiency and HIV-related enteropathy. The most common misdiagnosis is celiac disease as the malabsorption syndromes are similar, as are the endoscopic findings and small intestinal biopsies. ${ }^{1-3}$

In U.S., tropical sprue is commonly overlooked due to its much lower prevalence compared to celiac sprue. The treatment for tropical sprue begins with the replacement of folate, vitamin $\mathrm{B}_{12}$, iron and among other vitamins and nutrients. Tetracycline and trimethoprim/ sulfamethazole are recommended antibiotics for 3to6months. In children, tetracycline is avoided and other antibiotic regimens can be used. Malabsorption due to untreated tropical sprue can lead toimproper skeletal maturation and growth failure in children. ${ }^{1,3}$ Our patient is doing excellent after 6months of total therapy, and currently has no symptoms or any cognitive problems. This particular case demonstrates that tropical sprue still exists and can be observed in the Western countries due to immigration and worldwide travel.

\section{Acknowledgments}

None.

\section{Conflicts of interest}

Author declares there are no conflicts of interest.

\section{Funding}

None.

\section{References}

1. Brown IS, Bettington A, Bettington M, et al. Tropical sprue: revisiting an under recognized disease. Am J Surg Pathol. 2014;38(5):666-672.

2. Langenberg MC, Wismans PJ, van Genderen PJ. Distinguishing tropical sprue from celiac disease in returning travelers with chronic diarrhea: a diagnostic challenge. Travel Med Infect Dis. 2014;12(4):401-405.

3. Ghostal UC, Srivastava D, Verma A, et al. Tropical sprue in 2014: the new face of an old disease. Curr Gastroenterol Rep. 2014;16(6):391.

4. Datta Gupta S. Pathology of celiac disease: a brief review. Trop Gastroenterol. 2013;34(4):207-226. 